\title{
Increased RANKL/OPG ratio and sclerostin in patients with septic shock
}

\author{
Maria Bergquist $^{1 *}$, Sofia Andersson ${ }^{1}$, Christian Rylander ${ }^{2}$, Catharina Lindholm ${ }^{1}$ and Inger Gjertsson $^{1}$ \\ ${ }^{1}$ Department of Rheumatology and Inflammation Research, Sahlgrenska Academy, University of Gothenburg, Sweden \\ ${ }^{2}$ Department of Anesthesiology and Intensive Care Medicine, Institute of Clinical Sciences, Sahlgrenska Academy, University of Gothenburg, Gothenburg, Sweden
}

\begin{abstract}
We report an increased RANKL/OPG ratio and increased sclerostin levels in patients with septic shock one day after admission compared to healthy subjects, indicating an increased risk for bone loss in survivors of septic shock. No increase in RANKL/OPG ratio was observed in patients treated with hydrocortisone during septic shock.
\end{abstract}

\section{Background}

Patients recovered from critical illness have been demonstrated to have decreased bone mineral density (BMD) [1]. The global awareness of long term consequences of critical illness is rapidly increasing, but the identification of specific pathophysiological mechanisms is still tentative. Osteoporosis is widely considered an emerging health and socioeconomic threat contributing to morbidity and mortality in an ageing population [2]. The risk factors for secondary osteoporosis are well described and include (among others) female gender, high age, smoking and glucocorticoid treatment [3], but whether patients who recovered sepsis are at increased risk remains elusive.

Bone is formed by osteoblasts producing bone matrix proteins and mineral crystals [4]. Sclerostin is a glycoprotein secreted by osteocytes, inhibits bone formation of osteoblasts, and thus contributes to bone loss [5]. However, bone loss is essentially caused by osteoclasts resorbing bone matrix (mainly Collagen type I) through the release of proteolytic enzymes. The large multinucleated osteoclasts are generated by fusion of hematopoietic mononuclear osteoprogenitor cells, a process that requires activation of the Receptor Activator of NFKB (RANK) through its ligand (RANKL), which is expressed by osteoblasts, osteocytes, $\mathrm{T}$ and $B$ cells. This activation can be inhibited and RANKL neutralized by its decoy receptor osteoprotegerin (OPG) [4]. Thus, the RANKL/ OPG ratio is pivotal in the regulation of osteoclast differentiation, activation and survival and affects the balance between bone formation and resorption [6]. It has been shown that this ratio can be increased during inflammatory conditions, possibly caused by an increased release of cytokines [7]. However, to our knowledge it is not yet known if osteoclasts in vivo are affected by a severe infectious condition such as sepsis. The main aim of this study was to investigate the RANKL/OPG ratio in patients during and after septic shock.

\section{Patients and methods}

This multicenter study was approved by the Ethical Committee for Human Research in Uppsala, Sweden. Patients were recruited from a larger study cohort which has been described in a previous publication [8]. In short; inclusion criteria were (i) age over 18, (ii) sepsis according to the American-European consensus criteria [9] (iii) treatment with at least one vasopressor. Exclusion criteria were (i) known infection with human immunodeficiency virus or hepatitis B or C, (ii) chronic treatment with glucocorticoids (iii) participation in any investigational drug study within four weeks preceding the study period, (iv) survival expected to be shorter than three days. Informed consent was initially obtained via next of kin and later directly from survivors. Consenting healthy subjects were recruited among non-smoking laboratory and hospital staff without any chronic or acute illness and with no medication. Blood samples were collected during septic shock; at T0 within 24 hours of ICU admission, T1 24 hours after T0, T2 48-120 hours after T0. The timepoint T3 was sampled 4-19 days after ICU admission when the acute inflammatory phase was considered to have resolved and the patient was stable without support to vital functions. Finally, blood was sampled after recovery at T4, 5-13 months after the patient had been discharged from the ICU. Samples were centrifuged at $1500 \mathrm{~g}$ for 15 minutes and plasma was stored in $-70^{\circ} \mathrm{C}$ until analysis. For quantification of markers of bone resorption, plasma samples were analyzed using commercially available enzyme linked immunosorbent assay (ELISA) kits (sRANKL and Sclerostin (Cusabio, College Park, MD, USA) and OPG (eBioscience, San Diego, CA, USA)")" according to the manufacturer's recommendations. Optical density was read at $450 \mathrm{~nm}$ using a Spectra Max 340PC (Molecular Devices, Sunnyvale, California) running the SoftMax Pro 5.2 software. Data were graphically presented as group means and standard error of the mean (SEM). Differences between patients at T1 and healthy subjects and between patients treated with or not treated with hydrocortisone were tested with the two-tailed Mann-Whitney test (GaphPad Prism 6.0 for Windows, GraphPad Software Inc, La Jolla, CA, USA). A p value $<0.05$ was considered statistically significant.

Correspondence to: Maria Bergquist, Department of Rheumatology and Inflammation Research, Sahlgrenska Academy, University of Gothenburg, Sweden, E-mail: maria.bergquist@gu.se

Key words: septic shock, bone, RANKL, OPG, sclerostin, osteoclasts

Received: January 09, 2017; Accepted: January 30, 2017; Published: February 03,2017 
A

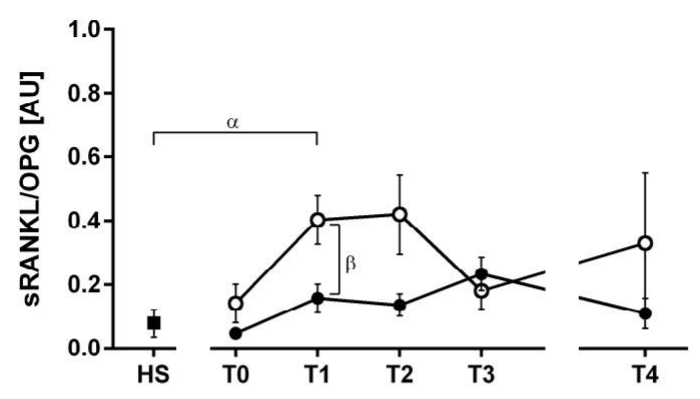

B

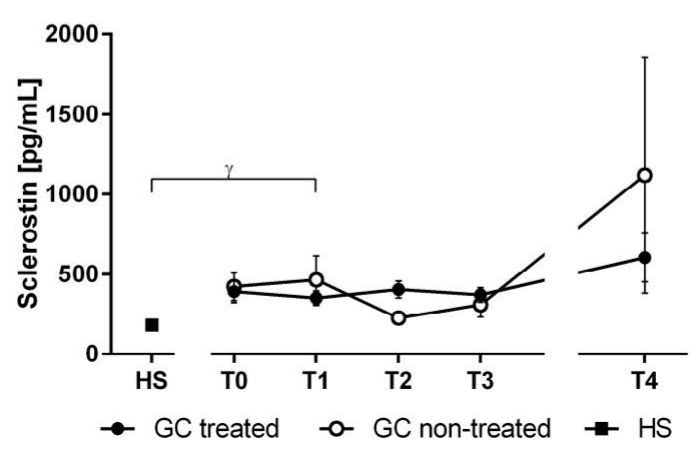

Figure 1. Ratio of receptor activator of nuclear factor kappa B (NFkB) ligand (RANKL) and osteoprotegerin (OPG) (A) and sclerostin (B) measured during the initial phase of septic shock (T0-T2), after the acute inflammatory phase (T3), after recovery (T4) and from healthy subjects (HS). Data represent means and standard deviation of the mean (SEM). Patients were divided in groups as glucocorticoid (GC) treated (filled circles) and non-treated (open circles). Differences between all patients and healthy subjects at $\mathrm{T} 1$ $(\alpha, \mathrm{p}=0.008$ and $\gamma, \mathrm{p}=0.003)$, and between GC treated and non-treated patients at T1 ( $\beta$, $\mathrm{p}=0.014$ ) were tested using two-tailed Mann-Whitney test.

\section{Results and discussion}

In this study we quantified markers of osteoclast activation, RANKL and OPG as well as sclerostin as a negative marker of bone formation, in 15 patients ( 8 males) with septic shock and 9 healthy subjects (4 males). The median (range) age of the patients and healthy subjects were $62(32-83)$ and 52 (32-67) respectively. We observed limited but consistent data suggesting increased osteoclastic activity and decreased bone formation in patients with septic shock compared to healthy subjects. During the day after admission (T1), the RANKL/ OPG ratio had increased in the patients compared to healthy subjects ( $\mathrm{p}=0.0083$ ), starting from a level similar to that of healthy subjects at T0 (Figure 1A). In most of the patients, the ratio increased further and peaked during the shock phase (T2). After recovery (T4), the ratio of most of the patients had retroceded to levels similar to healthy subjects. The concentration of sclerostin was increased in the patients compared to healthy subjects at $\mathrm{T} 1$ and did not retrocede to the levels of healthy subjects during the study period (Figure 1B). Our finding points to increased bone resorption, which is in broad agreement with previous studies observing increased concentrations of other bone resorption markers (urinary pyridinoline and deoxypyridinoline) in patients with sepsis compared to trauma [10] and surgery [11]. Sclerostin has previously been associated with bone loss in human diseases related to immobilization such as paralysis [12], but to our knowledge no reports have yet assessed the association between sclerostin and septic shock. The elevated ratio between RANKL/OPG in patients during septic shock is most likely associated with increased osteoclast activation, and the increased concentration of sclerostin points towards decreased bone formation. As sepsis is a multifactorial critical illness, the underlying mechanism contributing to the observed shift in bone metabolism may be attributed to several factors, there among immobilization, cytokine release and nitric oxide production [13]. Numerous proinflammatory cytokines are known to increase RANKL mRNA expression in human osteoblastic cells. In addition, many patients with septic shock are treated with hydrocortisone according to the current clinical guidelines [14]. Among the patients included in the present study, 9 of 15 were treated with hydrocortisone for septic shock at some point during intensive care. Interestingly, the patients treated with hydrocortisone displayed a decreased RANKL/OPG ratio compared to patients not treated with hydrocortisone $(\mathrm{p}=0.014)$. This finding stands in contrast to the well described adverse effects of high dose therapeutic glucocorticoids on bone as well as previous studies demonstrating that RANKL expression is upregulated by glucocorticoids in vitro [15]. However, increasing evidence suggests that the catabolic and anabolic effects on bone caused by glucocorticoids may be dose dependent [14]. The hydrocortisone dose used for treatment during septic shock is relatively low, meant to replace the physiological concentration of cortisol during septic shock. At such levels, glucocorticoids have been shown to cause mesenchymal progenitor cells to differentiate into osteoblasts, and thereby preserve and increase bone formation [14]. It is also possible that low dose glucocorticoid treatment may inhibit bone loss by dampening inflammation and proinflammatory cytokine release. If the finding of a decreased RANKL/OPG ratio in treated patients can be confirmed, glucocorticoid treatment may be useful in preserving bone formation in patients at risk of inflammation associated bone loss. However, as the concentration of sclerostin was unaffected by glucocorticoid treatment in these patients, a decrease in bone formation associated with septic shock may not be recovered by glucocorticoid treatment.

\section{Conclusions}

The increased ratio of RANKL/OPG and increased concentration of sclerostin suggest increased bone loss in patients with septic shock. These patients may be at elevated risk of osteoporosis following their critical illness, but further studies are needed to confirm this hypothesis. Although data are limited, we also observed a lower RANKL/OPG ratio in the patients treated with hydrocortisone compared to patients not treated with any glucocorticoids. Future studies should incorporate clinical measures of bone density to confirm whether RANKL/OPG ratio and sclerostin could be used as biomarkers for increased risk of inflammation associated osteoporosis in patients with septic shock.

\section{Conflict of interest}

This multicenter study was approved by the Ethical Committee for Human Research in Uppsala, Sweden. All authors have contributed significantly to the scientific work and the writing of this manuscript, and declare no conflict of interest.

\section{References}

1. Orford NR, Lane SE, Bailey M, Pasco JA, Cattigan C, et al. (2016) Changes in Bone Mineral Density in the Year after Critical Illness. Am J Resp Crit Care Med 193: 736-744.

2. Rachner TD, Khosla S, Hofbauer LC (2011) Osteoporosis: now and the future. Lancet 377: 1276-1287. [Crossref]

3. Nguyen TV, Center JR, Eisman JA (2004) Osteoporosis: underrated, underdiagnosed and undertreated. Med J Aust 180: S18-22. [Crossref]

4. Lorenzo J, Horowitz M, Choi Y (2008) Osteoimmunology: interactions of the bone and 
immune system. Endocr Rev 29: 403-440. [Crossref]

5. Compton JT, Lee FY (2014) A review of osteocyte function and the emerging importance of sclerostin. J Bone Joint Surg Am 96: 1659-1668. [Crossref]

6. Simonet WS, Lacey DL, Dunstan CR, Kelley M, Chang MS, et al. (1997) Osteoprotegerin: a novel secreted protein involved in the regulation of bone density. Cell 89: 309-319. [Crossref]

7. Weitzmann MN (2013) The Role of Inflammatory Cytokines, the RANKL/OPG Axis, and the Immunoskeletal Interface in Physiological Bone Turnover and Osteoporosis. Scientifica (Cairo) 2013: 125705. [Crossref]

8. Bergquist M, Lindholm C, Strinnholm M, Hedenstierna G, Rylander C (2015) Impairment of neutrophilic glucocorticoid receptor function in patients treated with steroids for septic shock. Intensive care Med 3: 59 .

9. Levy MM, Fink MP, Marshall JC, Abraham E, Angus D, et al. (2003) 2001 SCCM/ ESICM/ACCP/ATS/SIS International Sepsis Definitions Conference. Intensive Care Med 31: 1250-1256. [Crossref]

10. Smith LM, Cuthbertson B, Harvie J, Webster N, Robins S, et al. (2002) Increased bone resorption in the critically ill: association with sepsis and increased nitric oxide production. Crit Care Med 30: 837-840. [Crossref]

11. Lind L, Carlstedt F, Rastad J, Stiernström H, Stridsberg M, et al. (2000) Hypocalcemia and parathyroid hormone secretion in critically ill patients. Crit Care Med 28: 93-99. [Crossref]

12. Morse LR, Sudhakar S, Danilack V, Tun C, Lazzari A, et al. (2012) Association between sclerostin and bone density in chronic spinal cord injury. J Bone Mineral Res 27: 352-359. [Crossref].

13. Gowen M, MacDonald BR, Hughes DE, Skjodt H, Russell RG (1986) Immune cells and bone resorption. Adv Exp Med Biol 208: 261-273. [Crossref]

14. Zhou H, Cooper MS, Seibel MJ (2013) Endogenous Glucocorticoids and Bone. Bone Res 1: 107-119. [Crossref]

15. Hofbauer LC, Gori F, Riggs BL, Lacey DL, Dunstan CR, et al. (1999) Stimulation of osteoprotegerin ligand and inhibition of osteoprotegerin production by glucocorticoids in human osteoblastic lineage cells: potential paracrine mechanisms of glucocorticoidinduced osteoporosis. Endocrinology 140: 4382-4389. [Crossref]

Copyright: (C2017 Bergquist M. This is an open-access article distributed under the terms of the Creative Commons Attribution License, which permits unrestricted use, distribution, and reproduction in any medium, provided the original author and source are credited. 